\title{
Entropy at the Level of Individual Particles: Analysis of Maxwell's Agent with a Hidden-Variable Theory
}

\author{
Dirk J. Pons ${ }^{1}{ }^{*}$, Arion D. Pons ${ }^{2}$, Aiden J. Pons ${ }^{3}$ \\ ${ }^{1}$ Department of Mechanical Engineering, University of Canterbury, Christchurch, New Zealand \\ ${ }^{2}$ University of Cambridge, Cambridge, UK \\ ${ }^{3}$ Rangiora New Life School, Rangiora, New Zealand \\ Email: *dirk.pons@canterbury.ac.nz
}

Received 23 April 2016; accepted 26 June 2016; published 29 June 2016

Copyright (C) 2016 by authors and Scientific Research Publishing Inc.

This work is licensed under the Creative Commons Attribution International License (CC BY). http://creativecommons.org/licenses/by/4.0/

(c) (i) Open Access

\begin{abstract}
Problem: Maxwell's Agent (MA) is a thought experiment about whether the second Law is violated at smaller scales. This is a complex problem because the scale dependencies are unclear for perfect gas assumptions, quantum coherence, thermalisation, and contextual measurement. Purpose: The MA is explored from a non-local hidden-variable (NLHV) perspective. Approach: The Cordus theory, a specific NLHV solution, was applied at macroscopic to fundamental scales. Physical realism requires the Agent be included in the analysis. Findings: The primary function is sorting, i.e. a one-time separation of species by some attribute. The thermodynamic MA situation is merely a special case for reducing disorder (entropy). A one-time extraction of energy is possible. This requires input energy, hence the device only has thermodynamic leverage and is not a perpetual motion device. Inefficiencies arise from thermalisation causing short mean free path of Brownian motion, perfect gases having minimal interaction with the gate, ambiguity about spatial location arising from quantum superposition, contextual measurement interfering with the particle velocity, and bremsstrahlung hysteresis losses occurring when the Agent operates. Implications: Entropy is a group property at the bulk level, not a characteristic of the individual particle, and can be reversed at an energy cost at the particle level. Originality: The explanation spans multiple levels from macroscopic down to fundamental, which is unusual. Achieving an explanation from the NLHV sector is novel. The theory accommodates superposition, irreversibility, entropy, contextual measurement, coherence-discord transition, and Brownian motion.
\end{abstract}

\section{Keywords}

Entropy, Irreversibility, Ratchet, Demon, Thermodynamics, Contextual Measurement

\footnotetext{
*Corresponding author.
}

How to cite this paper: Pons, D.J., Pons, A.D. and Pons, A.J. (2016) Entropy at the Level of Individual Particles: Analysis of Maxwell's Agent with a Hidden-Variable Theory. Journal of Modern Physics, 7, 1277-1295. 


\section{Introduction}

Maxwell's Agent (MA) is a thought experiment about whether it is possible to violate the second Law of thermodynamics at the microscopic scale. Maxwell's original idea [1] was that the motions of gas molecules in a vessel were variable, as evident in Brownian motion, so that a microscopic being with sufficiently sharp senses might detect the faster gas molecules in a container, and momentarily open a door to selectively let them into a second chamber. This would cause the temperature to drop in the first vessel, and rise in the second. ${ }^{1}$ This is represented in Figure 1.

If this worked, it would appear to violate the second law because the temperature difference could subsequently be used to generate work, hence the paradoxical outcome of a perpetual motion machine. The essence of this is a process of measure -> decide -> act that sorts out the favourable outcomes. Other conceptual embodiments are the Brownian ratchet, and feedback engines.

The context was that Maxwell noted the second law applied statistically to bodies en-masse, and he questioned whether the law applied at the microscopic scale where the individual molecules became apparent. By implication he expected that the law could break down, that the being "would be able to do what is at present impossible to us". Technology is now approximately at the point where individual molecules can be sensed and controlled, so after many years of quiescence the MA is again a topic of interest [2]. However, there has arguably

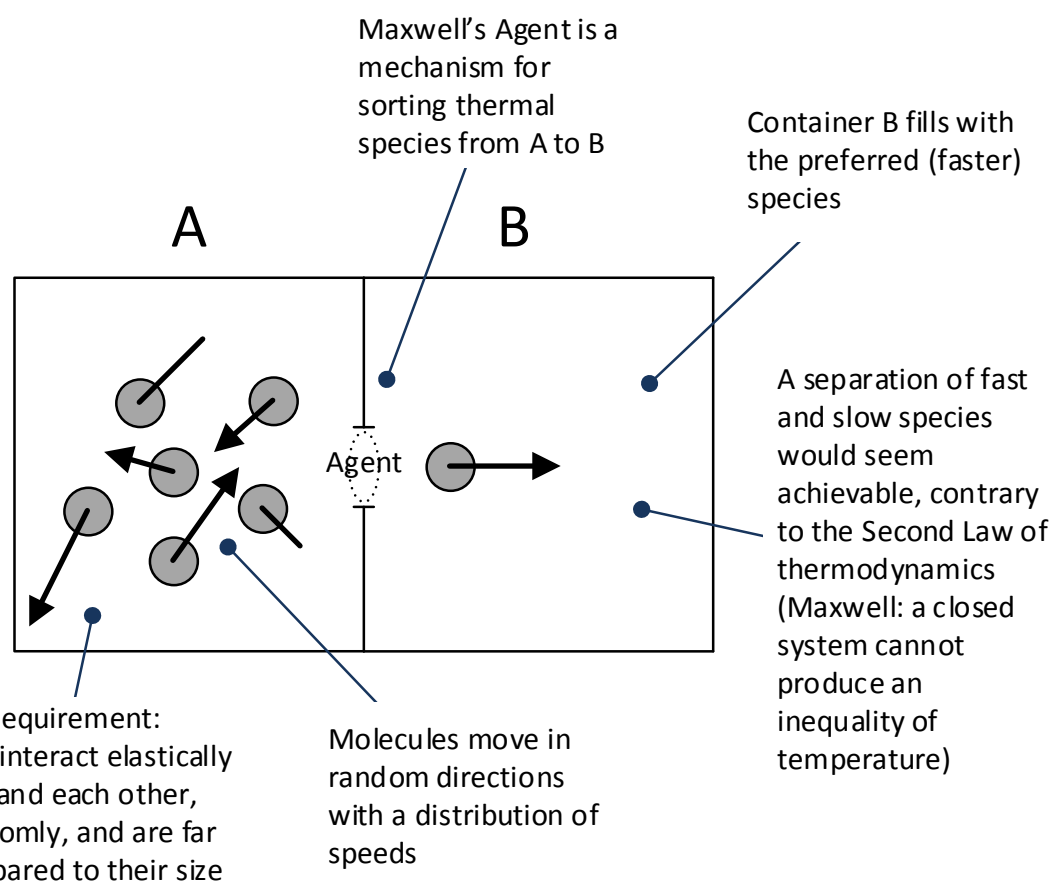

Ideal Gas Requirement: Molecules interact elastically with walls and each other, move randomly, and are far apart compared to their size
Maxwell's Agent is a

mechanism for

sorting thermal

species from A to B the preferred (faster) species

Figure 1. Maxwell's Agent is a thought experiment whereby the more energetic particles in vessel A are selectively admitted, by an active agent, into vessel $\mathrm{B}$. The central question is whether this would allow work to be extracted from the random motions of the particles in A. If so, that would imply a violation of the second law. However there is also the possibility, identified by Maxwell which the second law only applies to the fluid as a whole, not to the individual particles.

\footnotetext{
${ }^{1}$ Maxwell (1891) (p. 338-339): "One of the best established facts in Thermodynamics is that it is impossible in a system enclosed in an envelope which permits neither change of volume nor passage of heat, and in which both the temperature and the pressure are everywhere the same, to produce any inequality of temperature or of pressure without the expenditure of work. This is the second law of thermodynamics, and it is undoubtedly true as long as we can deal with bodies only in mass, and have no power of perceiving or handling the separate molecules of which they are made up. But if we conceive of a being whose faculties are so sharpened that he can follow every molecule in its course, such a being, whose attributes are as essentially finite as our own, would be able to do what is impossible to us. For we have seen that molecules in a vessel full of air at uniform temperature are moving with velocities by no means uniform, though the mean velocity of any great number of them, arbitrarily selected, is almost exactly uniform. Now let us suppose that such a vessel is divided into two portions, $\mathrm{A}$ and $\mathrm{B}$, by a division in which there is a small hole, and that a being, who can see the individual molecules, opens and closes this hole, so as to allow only the swifter molecules to pass from A to B, and only the slower molecules to pass from B to A. He will thus, without expenditure of work, raise the temperature of B and lower that of A, in contradiction to the second law of thermodynamics.”
} 
been no violation of the second law observed at these scales.

There are a number of questions: whether or not thermodynamics applies at the foundational level, whether or not a MA device could be built, and what the limitations of a real MA device might be. Maxwell's Agent does for the ontology of entropy what Schrodinger's Cat does for temporal superposition. The MA device is a thought experiment that provides a useful philosophical foil with which to probe the assumptions of thermodynamics at the fundamental level. Neither classical nor quantum perspectives of entropy definitively answer the question, so it is relevant to consider what the other sectors of physics make of the matter. These other sectors are primarily string theory- which has nothing specific to say on this topic and the non-local hidden-variable (NLHV) solutions-which do.

In keeping with the conjectural nature of Maxwell's question, the present paper treats the topic conceptually, using a thought experiment based on the NLHV solution provided by the prospective physics of Cordus theory. The NLHV theories all presume an internal structure at the sub-particle level, although such theories are exotic and not substantiated to the same degree as classical and quantum mechanics, neither are they frivolous. At times they have provided new insight into difficult problems (examples given below). Consequently it is worth evaluating Maxwell's question using new physics, for the ontological potential: doing this may suggest new ways of understanding the possible deeper causal mechanics of the second law of thermodynamics.

In keeping with the assumption of physical realism (that physical observable phenomena have deeper causal mechanics involving parameters that exist objectively and correspond to some physical features of the particle) that underpins NLHV theories, we use the term "Agent” to denote a physical mechanism, not necessarily either living or spiritual, with the ability to sense, make a decision, and perform a physical action, but not necessarily sentient. In comparison, Maxwell used the term “being”, whereas others later substituted “demon”.

\section{Existing Theoretical Explanations and Empirical Applications}

\subsection{Empirical Applications}

Macroscopic MA devices have occasionally been invented, but these are not perpetual motion machines, due to the effect of external fields or heat source and sinks, e.g. [3]. Practical applications of the MA exist, primarily as cooling [4] [5] or flow control [6] at the atomic level. However these devices require special setup including one-way barriers such as optical traps [7] [8], and external energy sources, thus do not raise thermodynamic paradoxes. The MA has been proposed as a mechanism in the vortex tube [9], though this too is not a perpetual motion effect, and there are other ways to explain that specific phenomenon. Other interesting applications of MA principles are in phase separation of grains in microgravity or vibrational suspension [10], and it has been proposed that the inelasticity of collisions causes clustering [11]. Molecular Brownian ratchets have also been proposed [12].

\subsection{Theoretical Explanations}

There are a number of theoretical explanations for why Maxwell’s agent should not work. Szilárd's explanation, which was a prominent early contribution, was that the agent has to do work to assess the velocity of the molecules. Thus including the agent as part of the system means that the agent provides the work, see [13]. Another explanation was that there is an information cost to measuring [14] [15], storing, or erasing information about the state of a molecule, and this information requires work [16]. This is a popular theoretical approach, and the many different theories all suggest that the energy needed to operate the MA will be at least as great as the work done [17]. Similarly, the work required to erase the information has been predicted to be as much gained by the thermal difference in the engine [18]. A more controversial explanation is that there are no faster and slower particles, they are all stationary [19] [20]. However, this is problematic as it is difficult to reconcile with observed Brownian motion.

Statistical simulation has been applied to better understand the MA behaviour in classical fluids (billiard-type systems). Examples include velocity changes between collisions [21], and contacts between billiard spaces with different types of voids which change the geometric location of the balls [22]. This type of work has also shown that the MA creates a density gradient rather than temperature per se [23].

Numerous theoretical attempts have been made to explain the effect using quantum theory, e.g. [24] [25]. The process, as applied to a Szilard engine, is then considered to involve 1) insertion of a barrier wall, 2) measure- 
ment, 3) expansion of the engine, and 4) removal of the barrier. Theoretical work suggests that the MA needs to know the number of entities involved in these condensates [26] [27], and that bosonic (as opposed to fermionic) condensates may give more work since the particles are identical [28]. Inverting the MA operation, it has been suggested that since measurement changes the energy of a quantum state, a MA could be used to power a heat engine via selective measurement [29]. However this idea does depend critically on the feasibility of making a reversible "pre-measurement”. It has been suggested that a superconductor tunnel junction acts as a MA, hence providing cooling ("Brownian refrigerator”) [30]. A limitation of the quantum approaches is the assumption that the working fluid is a coherent substance. This is evident in the many theories that depend on condensed substances and entangled states [31] [32]. This is a severe assumption since quantum coherence is not generally observed at the microscopic or macroscopic scales which Maxwell implicitly had in mind. Macroscopic states are characterised by discord (decoherence), which refers to non-superposition and non-entangled relationships between particles. This explicitly means that discord reduces the effectiveness of quantum-based MA mechanisms [33].

\subsection{Difficulties}

There are several obstacles to providing a complete scale-invariant explanation of the MA. One is the question of whether the perfect gas assumption is valid in the situation. Obviously real gases are imperfect, but how does this affect the MA outcome? Also, the question changes at a deeper level, since quantum mechanics assumes that particles are points. Another difficulty is how the Agent interacts with fields emitted by the particle. It is commonly stated that the Agent detects the energetic particles, but the methods of detection are seldom considered explicitly. The Agent cannot be permitted to generate photons and bounce them off the molecules, because that would involve adding energy to the system. So how does the detection occur, is it even possible to be non-intrusive from an energy perspective (c.f. Zeno effect), could it be lossless, and is it possible to use the fields emitted by the particle itself? Third, how to deal with the close-range thermalisation interactions that molecules make with each other in the moment between detection and admission? A simplistic assumption is that the whole operation of the Agent (detect-decide-open then close) operates instantly. However this is not physically possible since mass has to be moved and this takes time. Consequently the question is whether thermalisation invalidates the MA, and if so at what scales. Is there sufficient time between detection and action for the device to work? This is especially problematic if the particles have high velocity. How is quantum superposition to be included in the situation? Related to that is the difficulty of the transition from quantum to classical MA behaviour. Simple answers to these questions may be found by assuming that the Agent is outside the system, because then the Agent can be assigned powers that are not constrained by physical considerations. However such solutions are simplistic because they merely transfer part of the problem into the metaphysics domain.

Maxwell's question related to whether the thermodynamics at the foundational level are any different to those at the macroscopic, and that question is still incompletely answered. This is a complex problem because the scale dependencies are unclear for perfect gas assumptions, quantum coherence, thermalisation, and contextual measurement.

There is a need for analyses of the MA that 1 ) are based in physical realism (avoid resorting to metaphysical causation), and 2) span the scale from the macroscopic to the continuum represented by conventional thermodynamics, through to the level where particles display quantum properties, and potentially beyond to deeper physics at the sub-particle level. This is a challenging problem.

\section{Purpose and Method}

\subsection{Purpose}

The purpose of this paper is to explore the fundamental thermodynamic principles underpinning Maxwell's Agent. Specifically, we are interested in seeking insights from the hidden-variable sector. This sector has made little to no contribution to thermodynamics.

\subsection{Methodology}

Hidden-variable designs propose that matter particles have internal structures, the "hidden” variables. This is an intuitively attractive idea [34], but the difficulty has been finding suitable internal designs. Note that while the 
Bell-type inequalities [35] [36] preclude local hidden-variable solutions, they do not eliminate non-local designs. Historically the only hidden variable theory of substance has been the de Broglie-Bohm pilot wave theory [37] [38], but that has not developed into a wider theory of physics. The recent development of the Cordus theory [39] offers another candidate solution, and this is applied here. The present paper uses Maxwell's Agent to explore how thermodynamics scale from the macroscopic to fundamental levels under the assumptions of this NLHV theory. The area under examination is Maxwell's simple gated two-chamber arrangement.

The conjectured Cordus theory is a conceptual physics. It started as a designed solution to wave-particle duality in the double-slit device [39]. By design is meant that the internal structures of the particle (internal form) were determined by what was necessary and sufficient to describe the empirically observed behaviour (external functionality) of the photon in the double-slit device. The theory was built on the assumption of physical realism, and that physically observable attributes, such as spin, correspond to some physical feature of the particle. In contrast quantum mechanics assumes that particles are structure-less zero-dimensional (0-D) points and their properties, such as spin, are mathematical properties or intrinsic variables. The theory has subsequently been expanded to include other areas including photon emission [40], matter-antimatter annihilation [41], neutrino attributes and beta decay processes [42], structure of the atomic nucleus [43], pair production [44], and asymmetrical baryogenesis [45].

\subsection{Approach}

Consistent with the position of physical realism, Maxwell's "being" is required to be a physical Agent: whether alive or inanimate it must be made of matter and operate according to physics, and may not have paranormal or metaphysical capabilities. The purpose of this paper concerns the intersection of thermodynamics with fundamental physics and consequently there is no value in permitting the MA mechanism to operate beyond time, matter, fields, and space. This is relevant to note since the MA problem is often framed by assuming a priori that the agent must have the ability to sort particles, without actually considering how it does that, and then looking at the logical consequences of having such an ability. Our position is that the Agent is an integral part of the system, and its capabilities must be included in the analysis from the outset.

The approach was to apply the gedanken experiment method. This method takes a given set of principles of physics, which are the starting lemmas. The starting point could be any theory of physics: continuum mechanics, quantum mechanics, string theory, NLHV theory, etc. In this case it was the Cordus theory. The method then applied logical inference on those lemmas, thereby predicting the physical causality for other parts of the problem not originally explicitly covered by the lemmas. Thus the process extended the theory beyond its original lemmas. The implications of this theoretical extension were then reconciled against known phenomena, and novel insights formulated into new explanations.

The operation of the Agent was considered at multiple scales. The first was the macroscopic level, where we imagine the interaction of perfectly elastic balls. The next was the level of molecules, at which the perfect-gas behaviour is examined. The third scale was that of the particle level of quantum mechanics. The fourth was at the Cordus level, to assess the foundational thermodynamic implications for this theory.

\section{Results}

\subsection{Macroscopic Level: Thermodynamic Leverage and Sorting}

First, consider the capabilities of the Agent at the macroscopic level, with large vessels and perfectly elastic tennis balls. This is not usually the level at which thermodynamics is applied, but it is fruitful to start at this level as there are scale issues that become apparent later. Assume that the velocities of the balls are subject to Newtonian mechanics, specifically that balls do not change their velocities for no reason.

The Agent has to determine the velocity of each ball in vessel A before deciding whether to admit it to vessel B. Assume, in the macroscopic case of the balls, that the Agent is permitted to use photons for measuring the velocity of each ball, e.g. radar, because the energy of the photons is negligible compared to that of the targets. Based on the information received the Agent may then open the gate selectively, and thus capture the faster balls. Generating and using photons adds energy to the system. Even so, this small work associated with measurement would seem, at least in some macroscopic cases, to be much less than the kinetic energy gained by admitting to the vessel B the ball with its large kinetic energy. 


\subsubsection{Thermodynamic Leverage}

A once-off extraction of energy ex vessel B is then conceptually feasible. For example, the energetic balls in B could be made to strike a moveable plate, thereby performing work, see Figure 2. However, once this has been done, the energy states of vessels A and B would be the same, and there is no further energy that can be extracted. An alternative explanation is that once all the energetic balls have been sorted into vessel $\mathrm{B}$, leaving the slower ones in A, there is nothing more for the Agent to do. Both approaches show that an infinite extraction of energy is not possible.

There are some candidate exemptions. If vessel A were infinitely large then the process could be sustained. However that requires two infinite volumes: one infinite volume A from which to extract fast species, another working space $B$ in which to place them, and a third infinite volume $C$ in which to dump the spent material after its energy is extracted. So this is not realistic. Another exemption would be to have finite volumes, and extract work as before, but heat the waste material from B before reintroducing it to A. However that makes the MA nothing more than a refrigeration circuit, and there is no net work extracted.

Even if the energetic balls in B are returned to A after harvesting their energy, as shown in Figure 3, this will not bring about a perpetual energy device. Once their energy is extracted the balls become slow, and adding them back to A causes them to be of no further thermodynamic usefulness.

A practical implementation of a macroscopic MA device seems feasible, using large balls, sparsely distributed, moving no faster than the Agent can respond. However one would need to overlook the energy cost of running the Agent: the measurement, decision-making, and gate-opening. At the macroscopic level that cost can be comparatively small relative to the large energy of the balls. Even so, this analysis suggests that even a perfect MA mechanism with perfectly elastic collisions between macroscopic balls would be unable to function as a perpetual energy device. Instead a perfect Agent operating at macroscopic level merely provides good thermodynamic leverage: the exertion of a small amount of energy in measurement and computing allows a large decrease in entropy and the extraction, though only once, of work. In summary:

A practical implementation of a macroscopic MA device seems feasible, using large objects that are sparsely distributed. A macroscopic MA device permits, at best, a one-time extraction of energy. This is at the cost of input energy or parasitic losses, hence thermodynamic leverage. This would not be a perpetual energy device.

\subsubsection{Primary Macroscopic Outcome Is Sorting}

The MA is often portrayed as a mechanism for perpetual motion. However, the present analysis shows that energy cannot be endlessly harvested, but rather that mixtures can be separated albeit with some energy operating cost. The Agent achieves sorting, i.e. a one-time separation of species by some attribute. That attribute is

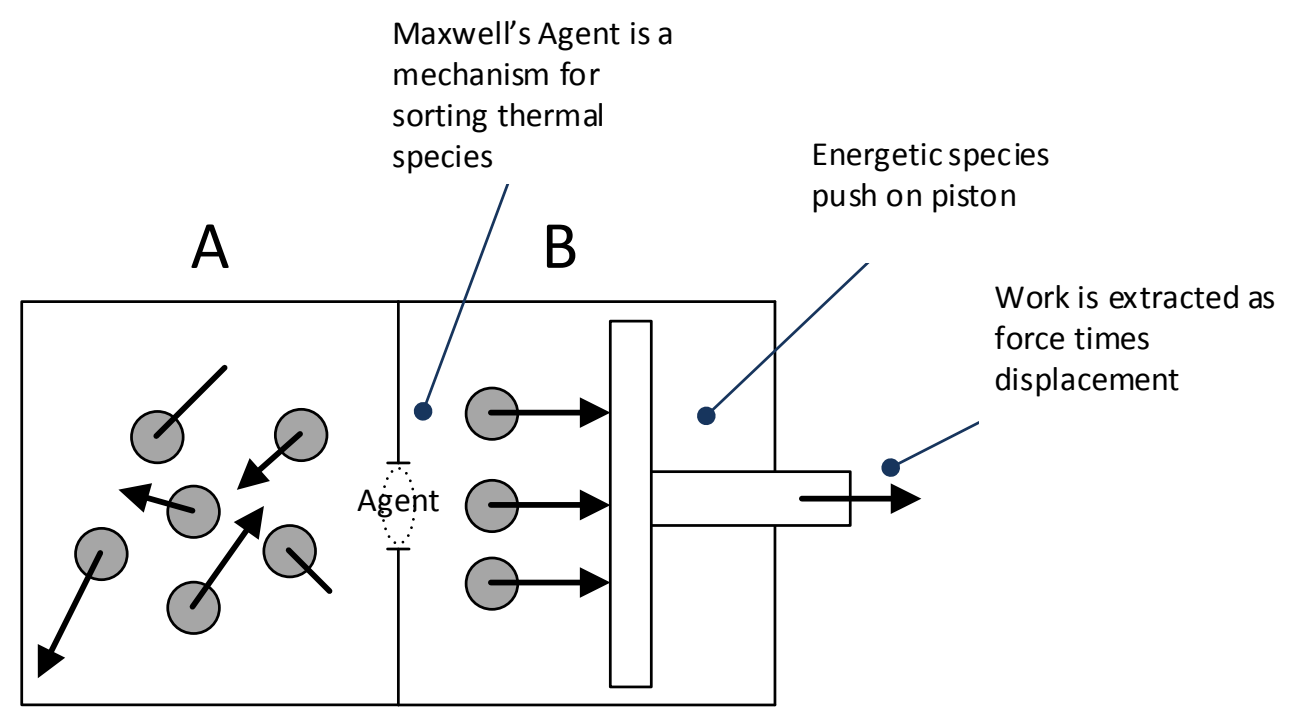

Figure 2. A once-off extraction of energy is feasible, if the Agent can sort out the more energetic species. The more captured energetic objects could, for example, be used to push a piston against an external resistance, thereby doing work. 


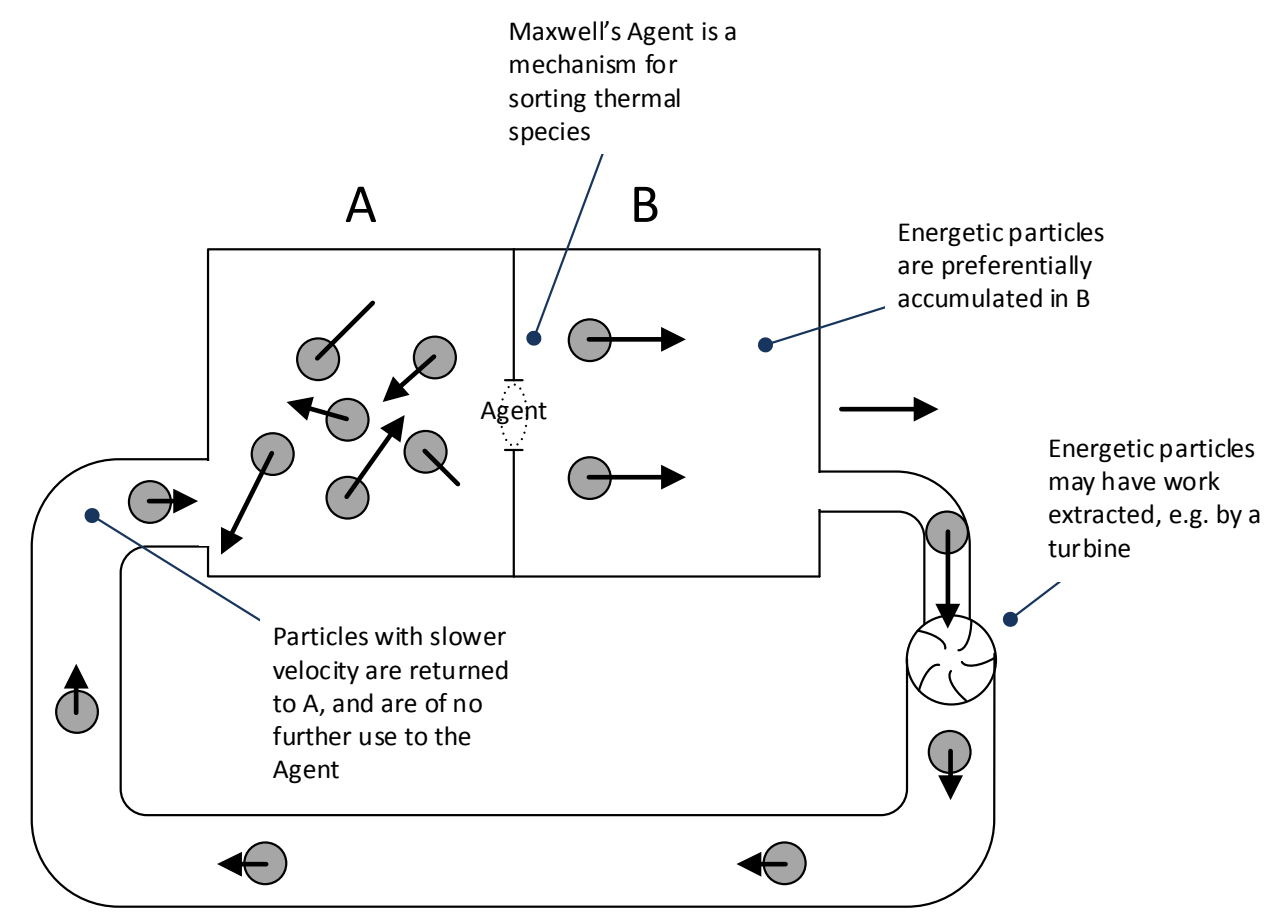

Figure 3. Extraction of energy is a one-time event. Return of the particles back to A causes the temperature to reduce in A, thereby limiting further energy extraction, even for a perfect Agent. So this is not a perpetual motion machine, though it may permit a one-time extraction of energy.

conventionally taken to be velocity, hence temperature in the vessels, and thus the problem is portrayed as a thermodynamic one. However the sorting could be by some other attribute, and at the macroscopic level this might be size, colour, charge etc. In such cases the problem is no longer primarily a thermodynamic one, but a reduction of disorder. In this context disorder refers to the degree of spread in the stochastic mixture of attributes: the MA reduces this spread by selective removal of objects with attributes within a certain range. In summary:

The primary function of a MA device is sorting and reducing disorder, i.e. a one-time separation of species by some attribute. The thermodynamic MA situation is merely a special case for sorting and reducing disorder, hence reduction of entropy.

Additional obstacles emerge that prevent an Agent from being perfect, and these become apparent at deeper levels of analysis.

\subsection{Microscopic Level: Gas Interactions at the Molecular Level}

Moving to the microscopic level, consider a fluid of perfect gas molecules. If we temporarily assume a perfect Agent-later we elaborate on the many imperfections and losses that a real Agent must have - then it is apparent that the same considerations apply as for the macroscopic balls: the Agent would function as a sorter of species and a reducer of disorder. If that sorting was by molecular velocity, then the outcomes would be thermodynamic, in the form of a once-off extraction of energy. The recycling of spent molecules back to vessel A would lower the temperature therein. Consequently, any energy that could be extracted would be limited by the temperatures of the vessels, hence the Carnot principle applies, and there is predicted to be no perpetual energy MA device at the molecular level (perfect gas) for vessels of finite volume. That is the optimistic loss-less situation and in reality there are more serious difficulties that the Agent must overcome, as follow.

\subsubsection{Need for External Power to Operate the Agent}

While the macroscopic balls may be detected with photons, we cannot permit this to be applied to the molecular scale because: 1) the momentum of the molecule may be appreciably affected, and 2) a larger number of photons are required to cope with the higher density of molecules, hence more work input. In this situation the energy 
cost of measurement could be great compared to the work gained, i.e. poor thermodynamic leverage. Sensing the molecules will have to be done by detecting their fields, and additional problems arise (see below).

\subsubsection{Momentum Transfer Eliminates the Difference in Velocity}

The next difficulty for the Agent is that momentum transfer between molecules erases their velocity difference before the Agent can act to separate them. This also applies in the macroscopic case of elastic balls, but is less severe. At the microscopic level of gas molecules the process is termed thermalisation, whereby the interaction between particles causes equal distribution of energy, hence thermal equilibrium of the gas. A gas can still have Brownian motion even after thermalisation has occurred, as Maxwell pointed out. Increasing the density of the macroscopic balls or microscopic molecules (objects) makes things worse, because although the Agent has more candidate objects within a given time, the objects also have more opportunity to collide with each other and thereby make the distribution of kinetic energy more homogenous. Decreasing the density of the objects might help the situation. For example, if there is only one fast and one slow object in vessel A, then the Agent could hope to capture the fast one eventually, regardless of which was carrying the momentum. (Two objects of equal mass will swap momentum at impact). So in the simplest situation the principle works.

However as more interacting objects are added the momentum transfers become more complex. It is one of the assumptions of an ideal gas that collisions between the gas particles occur much more often than collisions with the wall. Thus the assumption of an ideal gas maximally works against the Agent: the gas molecules have maximal interaction to equalise their momenta, and they contact the wall relatively infrequently. So the Agent, which of necessity is positioned at the wall, will see relatively few molecules, and these will tend to be homogeneous in velocity.

A contrary argument is that thermalisation is a bulk process and merely results in homogeneous average momentum, whereas there is a degree of random variability for each individual molecule, hence Brownian motion, that the Agent might still harvest. However this will not do either, for reasons of time, as we come to next.

\subsubsection{Time Is a Constraint on Efficiency}

The difficulty for an Agent that seeks to sort based on random or Brownian motion is that the trajectory of any one molecule cannot be predicted until it is right at the wall, by which time it may be too late to capture it for vessel B. The Agent is static and must wait at its gate for an opportune moment when a high-velocity object happens to be naturally headed towards the gate. Thus the Agent needs time, in the form of waiting for a chance event, plus the time for measurement and capture. The same time provides a gap between the moment of detection and the moment of capture, and this time also allows the molecules to transfer momentum between each other at collisions, and thereby change or erase the initially measured velocity. The greater the particle density (number of participating molecules or objects per unit volume), the greater the momentum transfer opportunities, and the worse the problem for the Agent.

\subsubsection{Mean Free Path and Operational Depth}

For an Agent attempting to harness random or Brownian motion, it is preferable that the mean free path of the molecules is large. However circumstances do not favour this. The path length, i.e. distance between collisions, is inversely proportional to the product of the density (or pressure) and square of the particle diameter. This is problematic because the MA device prefers a denser gas (or higher pressure) with its greater energy density. The mean free path is of the order $0.1 \mathrm{um}$ at $100 \mathrm{kPa}, 373 \mathrm{~K}$, for molecules of $3 \mathrm{E}-10$ m diameter. $^{2}$

We therefore introduce the concept that an Agent has an operational depth, this being the distance from the point where objects are detected, to vessel B. In the case of gas molecules this depth must be shorter than the mean free path of the molecules, so that the Agent may successfully complete all its detection and capture processes before the molecule changes velocity or direction. However the operational depth cannot be made arbitrarily short, because it depends on the time required to detect velocity (speed and direction), time for computation \& decision-making, time to open and close the gate. Each of these requires other matter particles to be moved and then reset, hence the expenditure of energy and the consumption of time. While the operation of the Agent might be accelerated (e.g. faster electronics) to reduce the operational depth, the speed of light imposes a fundamental limit. Thus the further obstacle to constructing a working MA device is the necessity for fast operation, which is a challenge for implementation.

${ }^{2}$ http://hyperphysics.phy-astr.gsu.edu/hbase/kinetic/menfre.html\#c3. 
A further problem is that a short mean free path results in information about a molecule quickly becoming obsolete, and hence requires the Agent to undertake frequent re-measurement activities. These have an energy cost, which will need to be supplied externally or met by the harvested molecule.

The lower the particle density, in the extreme case only two objects in the vessel, the less the momentum transfer, but the longer the Agent must wait for a fortuitous alignment with the gate. If the Agent is expending energy in maintaining vigilant detection, then the temporal summation thereof must be offset against the once-off energy extraction possible from the system. This does not make for an easy energy breakeven point: the objects need have a certain minimum momentum to make the wait worthwhile. Also, it is preferable that the objects have high mass rather than high velocity. This is because higher velocity requires more frequent monitoring by the Agent and therefore more energy usage. In summary:

Practical difficulties exist for the Agent when operating on a molecular fluid, in that thermalisation erases the velocity difference between objects, such that the residual Brownian motion has a short mean free path. The Agent needs time to wait for a fortuitous incoming energetic object. However that same time also permits more thermalisation between the objects. This requires the Agent to undertake frequent re-measurement activities, with associated energy cost. The perfect gas assumption is incompatible with the Agent as it reduces the interaction of the objects with the wall. The larger the objects, and the fewer, the less the thermalisation and the greater the thermodynamic leverage, but the longer the Agent must wait for a fortuitous alignment with the gate. As the objects become smaller in momenta and more numerous, so the thermodynamic leverage decreases, and in the limit becomes zero.

\subsection{Quantum Mechanics Level: Zero-Dimensional Point Particles}

Assume that the MA device is filled with an electron gas as the fluid. Under quantum theory, a subatomic particle like the electron is not a single point but rather exists in two positions at once, hence quantum superposition. What does this mean for the MA device? The existing literature in this area has been summarised above, and here some additional comments are made.

\subsubsection{Superposition and Uncertainty}

The quantum mechanics perspective is that the electron is in geometric superposition, i.e. simultaneously in two locations in space, or more precisely its existence is stochastically distributed around two locations. QM asserts that the location is fundamentally uncertain and driven only by a probability function, the wave function. This also means that the exact position of the electron is fundamentally unknowable. Obviously this will cause difficulty for the Agent.

\subsubsection{Measurement}

Measurement is a key part of the activities of the Agent. Empirical evidence, e.g. the Zeno effect, shows that measurement affects the system under examination and that the process of measurement does affect a system in superposition. However it is difficult to explain how the act of observation causes the wave-function to collapse and the system to take a specific value, which is the problem of contextual measurement. Also problematic is that QM explanations of the MA assume that particles are in a quantum (coherent) state, but this is not the empirically observed behaviour at the macroscopic scale. It is difficult to explain why and where the transition occurs between coherent and discoherent states. Consequently the QM explanations of the MA do not cover the full scale from fundamental to macroscopic.

\subsubsection{Pre-Existing Properties or Not?}

Another difficulty for the MA device is that apply quantum mechanics denies that particles have pre-existing values. Per the Heisenberg uncertainty principle, the position and velocity of the electron are not both simultaneously knowable to precision. So there is nothing definite for the Agent to work on, unless it expends energy making measurements that collapse the wave function. QM proposes that the intrinsic parameters of the electron, e.g. the frequency (hence energy), position, and velocity, do not exist until they are observed, hence that the act of measurement is what forces them to take specific values. In many empirical tests these problems are circumvented by using large ensembles of particles, some of which are measured sacrificially as representatives of the larger population. The resulting means and probability distributions for these ensembles are key input variables 
in the quantum theory. However, this does not definitively address the behaviour of individual particles.

\subsubsection{Quantum Mechanics at the MA Level}

It is difficult to see how the Agent might operate at the quantum level. For the sorting process the agent needs to know the velocity of the electron, and for the gating function it needs to know its position. Quantum mechanics does not permit both these to be known.

Most of the quantum analyses of the MA device in the literature have made the unrealistic assumption that the working fluid is a coherent substance, i.e. a condensed or entangled state [31] [32]. This is not how real fluids operate at macroscopic scales, and not the thermodynamic regime Maxwell had in mind. Consequently there is no conclusive explanation of the MA device at the QM level. In summary:

From a quantum mechanics perspective the Agent cannot know with certainty the energy, position, and velocity of the objects, due to Heisenberg uncertainty. This would limit the efficacy of the agent, and may be sufficient to altogether prevent it operating.

\subsection{Sub-Particle Level: Cordus Theory Applied to Maxwell’s Agent}

This section considers the level deeper than the 0-D particle of quantum theory, for which the Cordus theory is used. A brief summary of the theory is first provided to set the context.

The Cordus theory predicts a specific structure to matter. There is an inner structure comprising two reactive-ends, which are a small finite distance apart (span), and each behaves like a particle in its interaction with the external environment [39]. A fibril joins the reactive-ends and is a persistent and dynamic structure but does not interact with matter. It provides instantaneous connectivity and synchronicity between the two reactive-ends. There is also an external structure whereby the reactive-ends periodically energise at the de Broglie frequency, and in doing so emit discrete forces in one or more of three orthogonal directions into the external environment. These discrete forces functionally make up a flux line. As a whole this set of structures is called a particule where it is necessary to emphasise that this is not a 0-D point. See Figure 4 for an example.

Electric charge is carried at $1 / 3$ charge per discrete force, with the sign of the charge being determined by the direction of the discrete force. So the number and propagation direction of energised flux tubes determines the overall electric charge of the particule.

The stochastic nature of the QM superposition can be conceptually recovered, being understood to represent the energisation states of the two reactive ends. In QM the 0-D particle is in both places at once, and simultaneously in neither until observed. In the Cordus theory the particule oscillates its energised location between its two reactive ends. Superposition arises because the Cordus particule has two reactive-ends with a separation between them, and these energise in turn at a frequency. The act of observation forces the photon particule to collapse to one location.

\subsubsection{Agent with Cordus Particules}

Next, consider the MA from the perspective of the Cordus theory. There are several issues to consider.

Escape mechanisms

Each incoming electron has a velocity as a whole, but its location oscillates between two moving places. The gate will need to be large enough in section to accommodate the electron span. In addition, the Agent will need to measure the locations of both reactive-ends, so that it can keep the gate open for the necessary temporal window to capture both. Otherwise the electron may escape again by tunnelling (see below).

However this temporal window also means there is risk of escape by a previous captive while the gate is open. Hence the efficacy of the system is reduced. There is nothing in the formulation of the MA device that prevents backflow through the gate. Only if particles were classical 0D points would this temporal window be infinitesimally short. In summary:

For a working fluid comprising sub-microscopic particules, geometric superposition creates an ambiguity about the spatial location of the particule relative to the gate, which decreases the efficacy of the capture process.

\subsubsection{Tunnelling}

Superposition causes a further difficulty, in that at small scales it can be difficult to confine an electron. This is 


\section{Electron e}

Characterised by one discrete force in each of the three directions. Therefore this a highly stable structure.

The discrete forces are released rather than retained as in the photon. Consequently there is an enduring succession of discrete forces in each of the three directions, which creates a long-ranged force effect.

New discrete forces continue to be created and sent down the flux tube (hyff) at each frequency cycle Inner Fibril provides instantaneous communication between reactive ends

Type of reactive end: pulsatile. One reactive end energising and the other de-energising $\left(180^{\circ}\right.$ out of phase)

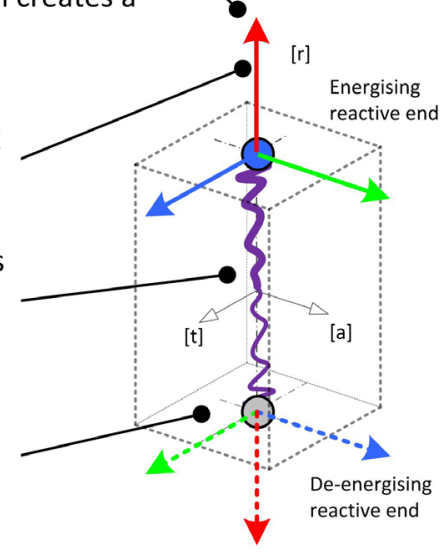

The HED notation represents the distribution of the discrete forces in the three emission directions (HEDs)

\section{HED notation}

Three orthogonal axes $(r, a, t)$ for emission of discrete forces

Dexter hand of energisation sequence for matter: red $\rightarrow$ green $\rightarrow$ blue. For the energising end this is $[\mathrm{r}] \rightarrow[\mathrm{a}] \rightarrow[\mathrm{t}]$.

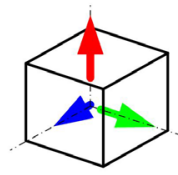

Each discrete force carries

$$
\text { (1) }
$$

Figure 4. The representation of the electron's internal and external structures. It is proposed that the particle has three orthogonal discrete forces, energised in turn at each reactive end. Adapted from [40] with permission.

evident in the empirically observed effects of the Josephson junction where a superconducting current can flow across a narrow insulating gap, and quantum tunnelling where a particle crosses an energy gap that should otherwise not be possible. The present theory has an explanation for tunnelling, which is given for photons at [46].

The Cordus theory anticipates that tunnelling would occur in the gate of the MA. Consider an electron with two reactive-ends in vessel A, and an Agent comprising a thin layer of material. Now the energised reactive-end emits discrete forces that interact with those of the Agent materials (e.g. the insulator atoms), hence generating reactive forces that prevent the reactive-end from penetrating the material, thus the electron is instead reflected. However the denergised reactive-end has low reactivity and can be displaced into the bulk of the Agent material, i.e. beyond the surface plane. If the denergised reactive-end starts to reenergise inside the bulk, then it is ejected back into medium A. However, if the thickness of the bulk material comprising the Agent is thin, then the denergised reactive-end may move through the thin barrier and then safely reenergise inside vessel $\mathrm{B}$. Then the other reactive-end may also travel through into vessel B at its next dormant cycle. Thus the particule as a whole can tunnel across the barrier. This is proposed as the explanation for the Josephson junction and quantum tunnelling. Unfortunately for the MA device, the electrons in vessel B can also tunnel back into A. So the MA de- 
vice needs a thin gate to reduce its operational depth relative to the mean free path of the objects, but tunnelling reduces the efficiency of a thin insulator. Only if particles are 0-D points would this not be a problem.

\subsubsection{Measurement at the Fundamental Scale}

Consider the measurement process of the Agent. Take a situation where vessel A is filled with an electron gas, with the electron as defined in the Cordus theory. Now consider the measurement responsibilities of the Agent at this fundamental level. It has to determine the velocity of each object (electron) before deciding whether to admit it to B. How will it do this? One option is for the Agent to generate photons and bounce them off the objects. Generating photons involves an energy cost for the Agent. The smaller the objects being measured, or the faster their velocity, the higher the required energy of the photon to obtain the necessary spatial and temporal resolution. This will adversely affect the efficiency of the Agent.

Furthermore there is still the question of how the photon might affect the electron. Specifically the photon may be absorbed by the electron, thereby changing its energy. This will be significant at the level of electrons and other small particules, less so at the macroscopic level of tennis balls. The Agent will then be pumping vessel A, and a strict MA implementation cannot permit this active measurement.

Are there ways of detecting the objects without the cost and interference of emitting photons at them? The answer to this depends on the philosophical position of whether objects have values that pre-exist, whether locality applies, and how contextual measurement works.

\subsubsection{Locality and Contextual Measurement}

The conventional theories of physics, including quantum mechanics, expect locality to be preserved. This means that a 0-D point particle is expected to be only affected by the values of the fields (electrical, magnetic, gravitational, etc.) at the infinitesimally small location of the point, a related expectation is that a property of the particle cannot be changed unless an external field or force is made to apply at that point. In contrast the Cordus theory proposes that the principle of locality fails. This is because the Cordus particule has a span and there are zones around each reactive-end where the discrete forces interact with the discrete forces of other particules. Consequently the particule is affected by more than the fields at its nominal centre point, and thus the theory asserts a Principle of Wider locality. Confirmatory evidence for this is seen in the evanescent field of the photon, whereby the locus of the photon is affected by material properties beyond its nominal point location [39].

The next issue is about the conceptuality of measurement, and what happens when an observer measures a quantum system. From the perspective of the Cordus theory there are two main ways to measure a particle. One is to collapse it, as when a photon is stopped and absorbed in a detector. This collapse provides information on its location. The theory proposes that measuring or otherwise grounding one reactive-end causes the whole photon to collapse [39]. The second method of measurement is by remote interaction through fields or flux tubes of discrete forces. This does not destroy the particle under examination, nor arrest it, but the interaction between the probing discrete forces and the particle's own emission if discrete forces affects its re-energisation and hence properties of location and energy (frequency). This has been applied to give a Cordus explanation of refraction and reflection of light [39], and the wider phenomenon includes the Zeno effect and the pumping of laser cavities. This second type of measurement is therefore always contextual: it depends on how the measurement is made and how aggressive the probing discrete forces are relative to the particle under examination. However, and this is important, any externally imposed measurement interaction will change the particle under examination, it is only a matter of degree.

Applying this to the MA situation means that it is impossible to assay the velocity of an electron, or any other object, without affecting it in some way. Even passively observing the proximity of an electron involves interacting with its discrete forces, hence interfering with it. Consequently, as the objects in the MA device become smaller, so the measurement intrusion becomes worse, and it becomes increasingly difficult to determine where the object is and how fast it is moving. Its location may be determined by arresting it, which is no use to the MA case. Or its velocity may be determined, but then the dynamic nature of the re-energisation process makes it difficult to be sure about the location of the current reactive-end. In this way the Cordus theory also conceptually recovers the Heisenberg uncertainty principle.

\subsubsection{Do Intrinsic Parameters Have Values Prior to Measurement?}

As previously identified, quantum theory proposes that objects do not have values that exist before measurement. 
In contrast the Cordus theory proposes that the electron does have specific values for all its parameters, that exist before the measurement takes place, but 1) they are dynamic and fast-changing (e.g. the position of the energising reactive-end can change so fast that it appears the particle is in two positions), 2) some of the parameters are coupled and cannot be measured simultaneously, and this is the uncertainty principle (e.g. the position and velocity of the electron are both determined by the progressive spatial position of the reactive-ends), 3) the act of measurement can interfere with the parameter and thereby change what is being measured (e.g. measurement of the frequency of a particle involves interacting with its discrete forces, hence affecting the emission thereof and thus affecting the frequency). In summary:

At the fundamental level, all measurement is contextual. It is impossible to assay the velocity of an object without affecting it in some way (contextual measurement intrusion). This applies even to passive observation of the fields of an object.

\subsubsection{Passive Detection of the Energetic Object}

So, no form of using photons to detect the incoming particules is acceptable at the fundamental level, if the objective is a thermodynamic extraction of energy. How about a less active form of measurement, like passively detecting the fields? Consider an Agent that waits passively and detects the electric fields of the approaching electrons, and assume that the sorting is by the energy (frequency) of the electron rather than velocity. Thus the Agent can wait until it detects a higher-than-average frequency, and then open the gate to admit that electron.

However, there are practical difficulties. There is the fundamental question of how the Agent would passively measure the field of an approaching electron. Assuming physical realism, it seems necessary that the Agent employ another charged particle to do this. This measurement particle could be another electron. The Agent would monitor the deflection of the measurement particle under the forces of the incoming particle, and respond accordingly. However this has further problems, as we shall see.

The next problem is that the Agent will need to determine the direction in which the electron is moving, since only electrons moving perpendicular to the wall, as opposed to tangential to it, are desired. Opening the gate to a tangential electron will not lead to capture, but may increase the chance of escapees from B. Determining the direction will need at least two measurements. Given the small mean path length in an equilibrated situation, this will be challenging: the measurement, decision-making, and gate-activation would need to be fast.

In an attempt to solve this, consider an improved trapdoor design of an Agent. Create a pore between vessels A and B, and block it with a molecule that includes a sensing electron. This sensor would move its position as it interacted with the fields of a sufficiently energetic incoming object, and this would cause the molecule as a whole to change shape and momentarily open the pore to let the energetic object through. Assume that the molecule could be designed so that it only hinged open for objects with velocity perpendicular to the wall. This design has the Agent simply responding opportunistically to energetic objects. It avoids all the memory issues of other MA proposals: the need to measure, record and erase data, and the energy cost thereof. Afterward the molecule of our hypothetical molecular Agent resets itself and repositions the sensor electron in readiness for another capture.

It might be thought that this restorative force could be elastic, e.g. mediated by bonds. Imagine a molecule that could be bent under the interaction with the incoming object, and then spring back into place when it had passed. We will also need to imagine that this molecule has some non-linear sensitivity such that it prevents back flow, for example that the sensor electron is the trigger rather than the gate itself, and is located in vessel A, hence insulated from B. It is like a one-way spring-loaded trapdoor that only lets through the energetic objects. We have to accept that our Agent may absorb some of the energy of the incoming object, but we can also anticipate that it would give the energy back to the object as it moved through the pore. Thus an Agent might appear not to require an energy source, as all its interactions would be elastic. However this is not so.

The measuring particle would need to be in a constrained location for deflection to be monitored, and would need a force to return it to the sensing position after a duty cycle. That resetting activity is problematic, because it causes hysteresis losses.

\subsubsection{Hysteresis Losses}

An elastic matter-based Agent using a charged particle (e.g. electron or proton) or even a neutron as the detector will have losses. This is because moving the sensing particle backwards and forwards involves acceleration, and it will emit a bremsstrahlung photon. Thus a hysteresis loss occurs. The same applies to the neutron, since it has 
a magnetic moment, albeit smaller. Even a perfectly balanced neutral particle with zero magnetic moment will still, per general relativity, emit a gravitational wave and radiate energy when subject to acceleration [47]. Although this emitted energy will be miniscule, it is nonetheless another loss that must be recouped from the harvested particle. The Agent requires external energy to reset so that it can continue operating. The Agent could be contrived to take the necessary energy from the objects that it captures, but that would defeat the purpose. It seems inevitable that a realistic design of Agent will always suffer from bremsstrahlung hysteresis losses. The losses might be reduced by moving matter more slowly, but this corresponds to an Agent that works slower. A slower Agent suffers from other problems, most especially the worsening of its ability to measure and complete a capture before energetic particules transfer their energy via impact with other particules. The converse is that the faster the Agent operates, the more likely it is to make a successful capture, but the greater the hysteresis losses.

Consequently we conclude that:

It is not possible to create a matter-based Agent that is lossless, because bremsstrahlung hysteresis losses will always occur when matter is moved back and forth within the Agent.

\subsubsection{A General Design of a Matter-Based Agent}

So we identify that the MA device requires energy to operate, and therefore cannot be lossless. However, if the energy it could extract from Brownian motion was sufficiently large, and it extracted this energy continuously, then this would not be a problem. This requires an examination of Brownian motion.

\subsection{Brownian Motion and Entropy per the Cordus Framework}

\subsubsection{Explanation of Entropy in Terms of Geometric Irreversibility}

The Cordus theory can be applied to explain Brownian motion. This is relevant since Brownian motion is what the MA device seeks to harness. Consider a particle P in vessel A of the MA device. The logic is as follows.

Particules have two ends, and hence two locations at which they can be affected. Each reactive-end emits discrete forces out into the external environment, contributing to a mesh of discrete forces in space, which is the fabric [48]. All the other particules do the same. Correspondingly, any particule $\mathrm{P}$ receives discrete forces from all the particules (many $\mathrm{N}$ ) in its accessible universe. In turn this fabric interferes with the emission of discrete forces for particule P. Due to the sizeable zone of influence provided by the Principle of Wider Locality, the particule $\mathrm{P}$ is affected by external fabric events in a zone outside the particule, including discrete forces in the fabric. These interfere with its own emissions, and therefore change its frequency. The interference also moves the location of the reactive-end in response to the imbalance between its own emitted discrete forces and the external discrete forces from the fabric. Since the fabric is discrete, it is also somewhat random in its composition. Consequently $\mathrm{P}$ will be buffeted by the fabric, and will be displaced one way and then the next. The movement of $\mathrm{P}$ means that at the next instant it is emitting discrete forces from a different location in the universe. Then the geometric superposition of the two reactive-ends means that disturbances at one reactive-end are communicated superluminally to the other, which then also engages with the fabric. This increases the complexity of the response of $\mathrm{P}$ to the fabric.

Consequently other remote particules $\mathrm{N}$ are also bumped into slightly different locations by the changed emissions of $\mathrm{P}$. The overall effect is that all the particules move around. The vast number of particules in the accessible universe, plus the time delay between them (fields are assumed to propagate only at the speed of light), adds so much complexity to the system that it is practically impossible to return particule $\mathrm{P}$ and all the many other particules $\mathrm{N}$ to their original geometric positions and energy states. Consequently the system as a whole is irreversible.

This, it is proposed, is where the arrow of time arises [49], and entropy too. This is because any original energy (or temperature) differences between objects in the fluid A are eventually dispersed among all the objects, and the irreversibility prevents the original state from being recovered. There is a chain of causality whereby the whole universe affects the position of the reactive-ends of particule $\mathrm{P}$, and as soon as $\mathrm{P}$ moves in response it starts to affect the whole universe in return. Thus the fluid in A becomes more thermodynamically homogenous, and energy becomes non-available for doing work. Hence entropy increases. In this context entropy represents all of the un-available energy, the disorder of the system, the number of ways the objects in A can be arranged, or the degree to which the system has deviated from the original geometric layout. 


\subsubsection{Brownian Motion}

Then Brownian motion may be explained in the Cordus theory as arising from the jostling by the fabric of many small particules P. These particules then apply forces to larger clumps of matter, such as molecules and collections of molecules, which aggregate those forces in unpredictable ways. Hence Brownian motion becomes observable at a macroscopic level, and does not require quantum coherence at this level. Larger objects are exposed to the fabric perturbations as much as smaller ones, but the Brownian motions of larger object are reduced because of the effects of cancellation of forces across the relatively rigid body, the many small forces receives, and larger inertial mass. Brownian movement is therefore proposed to be a proxy variable for a deeper fabric perturbation effect.

Accepting this interpretation that Brownian motion results from the interplay of discrete forces at the fundamental level, and adding the complexity of superposition and wider locality, then this suggests a further impediment to the successful operation of the MA device: the future path of an object changes rapidly and randomly. It is impossible to predict by the Agent. This worsens for smaller objects. Also, the more compact the Agent, the worse things get for its own efficiency (as above). In summary:

The random motions of small objects observed as Brownian motion, derive from the aggregation of multiple even finer random forces from the fabric, such that the future path of an object is unpredictable after it has been measured. This means there is no value in the Agent making a measurement of the object's position and velocity: both these will change before it moves through the gate. The smaller the object the greater the perturbation hence the greater the Brownian velocity but also the greater the variability.

The exception is if the object is already at the gate, and springs it open without the agent having to do any prior measurement. This also requires the gate to be very thin, so that the capture can be effected before the randomness of Brownian motion changes the direction of the object. However in that case objects in vessel $B$ will also be on the other side of the gate, and will escape or tunnel back to A. Such a device will not preserve a pressure or temperature differential: the two vessels will equilibrate. Even then the Agent will have hysteresis losses.

Thus the MA is predicted to be unable to extract work from Brownian motion. Thus in the end we find that the Maxwell Agent will not work at all at the fundamental level.

\section{Discussion}

\subsection{Outcomes}

This work provides several novel outcomes. The first is that it provides a comprehensive analysis of the Maxwell Agent apparatus, covering multiple levels from macroscopic to fundamental. The results predict that the MA device cannot be implemented as a perpetual motion device. It requires energy to operate. However it can in principle provide a one-time extraction of work for the expenditure of an initial energy. The analysis also shows that the MA device is primarily a sorting machine. It could in principle sort by attributes other than energy.

The argument is summarised as follows:

A practical implementation of a macroscopic MA device seems feasible, using large objects that are sparsely distributed. A macroscopic MA device permits, at best, a one-time extraction of energy. This is at the cost of input energy or parasitic losses, hence thermodynamic leverage. This would not be a perpetual energy device.

The primary function of a MA device is sorting and reducing disorder, i.e. a one-time separation of species by some attribute. The thermodynamic MA situation is merely a special case for sorting and reducing disorder, hence reduction of entropy.

Practical difficulties exist for the Agent when operating on a molecular fluid, in that thermalisation erases the velocity difference between objects, such that the residual Brownian motion has a short mean free path. The Agent needs time to wait for a fortuitous incoming energetic object. However that same time also permits more thermalisation between the objects. This requires the Agent to undertake frequent re-measurement activities, with associated energy cost. The perfect gas assumption is incompatible with the Agent as it reduces the interaction of the objects with the wall. The larger the objects, and the fewer, the less the thermalisation and the greater the thermodynamic leverage, but the longer the Agent must wait for a fortuitous alignment with the gate. As the objects become smaller in momenta and more numerous, so the thermodynamic leverage decreases, and in the limit becomes zero.

From a quantum mechanics perspective the Agent cannot know with certainty the energy, position, and veloc- 
ity of the objects, due to Heisenberg uncertainty. This would limit the efficacy of the agent, and may be sufficient to altogether prevent it operating.

For a working fluid comprising sub-microscopic particules, geometric superposition creates an ambiguity about the spatial location of the particule relative to the gate, which decreases the efficacy of the capture process.

At the fundamental level, all measurement is contextual. It is impossible to assay the velocity of an object without affecting it in some way (contextual measurement intrusion). This applies even to passive observation of the fields of an object.

It is not possible to create a matter-based Agent that is lossless, because bremsstrahlung hysteresis losses will always occur when matter is moved back and forth within the Agent.

The random motions of small objects observed as Brownian motion, derive from the aggregation of multiple even finer random forces from the fabric, such that the future path of an object is unpredictable after it has been measured. This means there is no value in the Agent making a measurement of the object's position and velocity: both these will change before it moves through the gate. The smaller the object the greater the perturbation hence the greater the Brownian velocity but also the greater the variability.

The exception is if the object is already at the gate, and springs it open without the agent having to do any prior measurement. This also requires the gate to be very thin, so that the capture can be effected before the randomness of Brownian motion changes the direction of the object. However in that case objects in vessel $B$ will also be on the other side of the gate, and will escape or tunnel back to A. Such a device will not preserve a pressure or temperature differential: the two vessels will equilibrate. Even then the Agent will have hysteresis losses.

The second novel contribution is that the paper shows that the NLHV solution of the Cordus theory can explain entropy and Brownian motion. The theory also provides physically natural explanations for superposition, entanglement, irreversibility, entropy, contextual measurement, coherence-discord transition, and tunnelling. The NLHV sector has otherwise not made significant contribution to these thermodynamic and related phenomena.

In this way we have offered a candidate explanation for the MA that is 1) based in physical realism and obviates the need for metaphysical mechanisms, and 2) covers the scale from the macroscopic to the quantum level and beyond. This is achieved with an original theory of physics. This theory conceptually recovers the continuum represented by conventional thermodynamics, and quantum behaviours. However it is not a derivation of quantum mechanics but rather a NLHV theory, and hence the complexity of the treatment provided here.

\subsection{Implications for the Second Law of Thermodynamics}

At the macroscopic level, the Second Law states that energy cannot be extracted from an equilibrated fluid, one with homogeneous pressure and velocity. Maxwell was questioning whether this still applied at the fundamental scale if there was some Agent to capture the particles with higher Brownian motion. The present analysis identifies that the Agent is primarily a sorting device and is energy-consuming, rather than purely a passive thermodynamic one-way device. Velocity is only one of the attributes that it might sort against. Whatever it sorts for, it always requires energy to operate, and this applies also to the thermodynamic situation. Depending on the application it might require more or less energy, and depending on the situation (object size, quantity, velocity) it might even give a once-off extraction of energy. However some energy consumption is inevitable even in the most passive designs conceivable, because of the need to move its matter-based working parts in space, hence low grade Bremsstrahlung emission and hysteresis losses. Consequently we dismiss the possibility that any physical Maxwell Agent could be a long-term perpetual energy device.

What about the extraction of energy from Brownian motion in an equilibrated fluid? The present analysis shows that the operating characteristics of the device become more compromised as the scale of the objects reduces from the macroscopic to the fundamental. The problems for the device are the necessity to use passive measurement (to avoid pumping the system) and the consequent difficulty of measuring the position and velocity of the objects. The latter issue is already problematic at the level of the perfect gas assumption, and becomes still more acute as the scale reduces further. When the situation is reduced to the scale at which Brownian motion is apparent, the analysis predicts that it is unlikely that even a once-off extraction of work will be possible.

Thus the present work finds in favour of the Second Law, by confirming that energy cannot be extracted from 
an equilibrated fluid at either macroscopic or particule level.

There are potentially interesting implications for entropy. Maxwell's paradox is only secondarily a thermodynamic question: it is really a question about sorting and hence entropy. The present analysis shows that the concept of entropy applies at the bulk level, and can be reversed (at an energy cost) at the particle level. Thus we have shown, using the Cordus NLHV theory, that entropy is a group property, i.e. an attribute of the assembly of particules, not a characteristic of the individual particle. This is close to Maxwell's original expectations. This also recovers the conventional statistical interpretation, but from a different direction.

\section{Conclusions}

The problem posed by Maxwell is a profound question about the intersection of thermodynamics with fundamental physics. This analysis shows that the NLHV design predicts that even a perfect MA mechanism with perfectly elastic collisions between macroscopic balls has the following limitations:

1) There is no perpetual energy MA device at either the macroscopic level (perfectly elastic ball collisions), or the molecular level (perfect gas).

2) The MA device is primarily a sorting machine, and thermodynamic sorting is merely one of multiple applications.

3) It permits at best a one-time extraction of energy from a mixture of energetic species, but only at large geometric scales. At small scales the opportunity for escapees increases, and the losses increase.

These conclusions are in accord with conventional analyses based on statistical mechanics. Consequently the predictions are not necessarily novel when looked in isolation. Rather, the original contribution is recovering these findings from the perspective of a single particle in a NLHV solution. This is valuable because it provides a rational explanation for the thermodynamic effects based on physical realism at the level of fundamental physics, which is not achieved by purely statistical or quantum approaches. Furthermore the same NLHV Cordus theory successfully explains or recovers many other phenomena including wave particle duality, basic optical laws of refraction \& reflection [39], pair production \& annihilation [44] [50], stability and instability of nuclides ( $\mathrm{H}$ to $\mathrm{Ne}$ ) [43] [51], asymmetrical lepto- \& baryogenesis [45], and the relativity of simultaneity (time dilation) [49]. The theory provides one coherent framework of explanation for all these phenomena. With the addition of the ability to explain aspects of thermodynamics at the fundamental level, the theory demonstrates resilience and validity.

The present work finds in favour of the second law, by confirming that energy cannot be extracted from an equilibrated fluid at either macroscopic or particule level, when applied over a longer time period. Further, entropy is a group property, not a characteristic of the individual particle. A MA is primarily a sorting device, and has the potential to change entropy, though with an energy cost.

\section{Acknowledgements}

The authors declare no funding sources external to their affiliations.

\section{Author Contributions}

All authors contributed to the creation of the underlying concept, development of the thermodynamic ideas, and editing of the paper. DP created the images and identified the hysteresis losses and Brownian motion.

\section{Conflict of Interest Statement}

The authors declare that there is no conflict of interests regarding the publication of this article. The research was conducted without personal financial benefit from any external funding body, nor did any such body influence the execution of the work or the decision to publish.

\section{References}

[1] Maxwell, J.C. (1891) Theory of Heat. Longmans, Green, and Co., London.

[2] Maruyama, K., Nori, F. and Vedral, V. (2009) Reviews of Modern Physics, 81, 1-23.

http://dx.doi.org/10.1103/RevModPhys.81.1 
[3] Wang, Y.-Z. (1986) Energy Conversion and Management, 26, 249-252. http://dx.doi.org/10.1016/0196-8904(86)90063-4

[4] Bannerman, S.T., Price, G.N., Viering, K. and Raizen, M.G. (2009) New Journal of Physics, 11, 63044.

[5] Binnewies, T., Sterr, U., Helmcke, J. and Riehle, F. (2000) Physical Review A-Atomic, Molecular, and Optical Physics, 62, 011601. http://dx.doi.org/10.1103/PhysRevA.62.011601

[6] Reinaudi, G. and Guery-Odelin, D. (2008) Physical Review A-Atomic, Molecular, and Optical Physics, 78, 015401.

[7] Thorn, J.J., Schoene, E.A., Li, T. and Steck, D.A. (2008) Physical Review Letters, 100, 240407. http://dx.doi.org/10.1103/PhysRevLett.100.240407

[8] Thorn, J.J., Schoene, E.A., Li, T. and Steck, D.A. (2009) Physical Review A-Atomic, Molecular, and Optical Physics, 79, 063402.

[9] Liew, R., Zeegers, J.C.H., Kuerten, J.G.M. and Michalek, W.R. (2012) Physical Review Letters, 109, 054503. http://dx.doi.org/10.1103/PhysRevLett.109.054503

[10] Eggers, J. (1999) Physical Review Letters, 83, 5322-5325. http://dx.doi.org/10.1103/PhysRevLett.83.5322

[11] Isert, N., Maa, C.C. and Aegerter, C.M. (2009) The European Physical Journal E, 28, 205-210. http://dx.doi.org/10.1140/epje/i2008-10403-7

[12] Chatterjee, M.N., Kay, E.R. and Leigh, D.A. (2006) Journal of the American Chemical Society, 128, 4058-4073. http://dx.doi.org/10.1021/ja057664z

[13] Quan, H.T., Liu, Y.-X., Sun, C.P. and Nori, F. (2007) Physical Review E-Statistical, Nonlinear, and Soft Matter Physics, 76, 031105. http://dx.doi.org/10.1103/PhysRevE.76.031105

[14] Vaikuntanathan, S. and Jarzynski, C. (2011) Physical Review E-Statistical, Nonlinear, and Soft Matter Physics, 83, 061120. http://dx.doi.org/10.1103/PhysRevE.83.061120

[15] Jacobs, K. (2012) Physical Review E—Statistical, Nonlinear, and Soft Matter Physics, 86, 040106. http://dx.doi.org/10.1103/PhysRevE.86.040106

[16] Moore, S.K. (2012) IEEE Spectrum, 49, 14-16. http://dx.doi.org/10.1109/mspec.2012.6189562

[17] Aquino, G., Grigolini, P. and Scafetta, N. (2001) Chaos, Solitons \& Fractals, 12, 2023-2038. http://dx.doi.org/10.1016/S0960-0779(00)00162-4

[18] Hosoya, A., Maruyama, K. and Shikano, Y. (2011) Physical Review E-Statistical, Nonlinear, and Soft Matter Physics, 84, 061117. http://dx.doi.org/10.1103/PhysRevE.84.061117

[19] Gyftopoulos, E.P. (2002) Physica A: Statistical Mechanics and Its Applications, 307, 421-436. http://dx.doi.org/10.1016/S0378-4371(01)00631-8

[20] Gyftopoulos, E.P. (2002) Physica A: Statistical Mechanics and Its Applications, 307, 405-420.

[21] Livorati, A.L.P., Loskutov, A. and Leonel, E.D. (2012) Physica A: Statistical Mechanics and Its Applications, 391, 4756-4762. http://dx.doi.org/10.1016/j.physa.2012.05.002

[22] Zaslavsky, G.M. and Edelman, M. (1997) Physical Review E-Statistical Physics, Plasmas, Fluids, and Related Interdisciplinary Topics, 56, 5310-5320. http://dx.doi.org/10.1103/physreve.56.5310

[23] Zheng, J., Zheng, X., Zhao, Y., Xie, Y., Yam, C., Chen, G., Jiang, Q. and Chwang, A.T. (2007) Physical Review E-Statistical, Nonlinear, and Soft Matter Physics, 75, 041109. http://dx.doi.org/10.1103/PhysRevE.75.041109

[24] Dong, H., Xu, D.Z., Cai, C.Y. and Sun, C.P. (2011) Physical Review E-Statistical, Nonlinear, and Soft Matter Physics, 83, 061108. http://dx.doi.org/10.1103/PhysRevE.83.061108

[25] Cai, C.Y., Dong, H. and Sun, C.P. (2012) Physical Review E-Statistical, Nonlinear, and Soft Matter Physics, 85, 031114. http://dx.doi.org/10.1103/PhysRevE.85.031114

[26] Niven, R.K. (2006) Physica A: Statistical Mechanics and Its Applications, 365, 142-149. http://dx.doi.org/10.1016/j.physa.2006.01.021

[27] Weiss, C. and Wilkens, M. (1997) Optics Express, 1, 272-283. http://dx.doi.org/10.1364/OE.1.000272

[28] Kim, S.W., Sagawa, T., De Liberato, S. and Ueda, M. (2011) Physical Review Letters, 106, 070401. http://dx.doi.org/10.1103/PhysRevLett.106.070401

[29] Erez, N. (2012) Physica Scripta, T151, 014028. http://dx.doi.org/10.1088/0031-8949/2012/t151/014028

[30] Pekola, J.P. and Hekking, F.W.J. (2007) Physical Review Letters, 98, 210604. http://dx.doi.org/10.1103/PhysRevLett.98.210604

[31] Jennings, D. and Rudolph, T. (2010) Physical Review E-Statistical, Nonlinear, and Soft Matter Physics, 81, 061130 
http://dx.doi.org/10.1103/PhysRevE.81.061130

[32] Maruyama, K., Morikoshi, F. and Vedral, V. (2005) Physical Review A-Atomic, Molecular, and Optical Physics, 71, 012108.

[33] Zurek, W.H. (2003) Physical Review A-Atomic, Molecular, and Optical Physics, 67, 012320.

[34] Einstein, A., Podolsky, B. and Rosen, N. (1935) Physical Review, 47, 777-780. http://dx.doi.org/10.1103/PhysRev.47.777

[35] Bell, J.S. (1964) Physics, 1, 195-200.

[36] Leggett, A. (2003) Foundations of Physics, 33, 1469-1493. http://dx.doi.org/10.1023/A:1026096313729

[37] De Broglie, L. (1925) Annales de Physique, 3, 98-109. http://tel.archives-ouvertes.fr/docs/00/04/70/78/PDF/tel-00006807.pdf

[38] Bohm, D. and Bub, J. (1966) Reviews of Modern Physics, 38, 453-469. http://dx.doi.org/10.1103/RevModPhys.38.453

[39] Pons, D.J., Pons, A.D., Pons, A.M. and Pons, A.J. (2012) Physics Essays, 25, 132-140. http://dx.doi.org/10.4006/0836-1398-25.1.132

[40] Pons, D.J. (2015) Applied Physics Research, 7, 14-26. http://dx.doi.org/10.5539/apr.v7n4p24

[41] Pons, D.J., Pons, A.D. and Pons, A.J. (2014) Physics Essays, 27, 26-35. http://dx.doi.org/10.4006/0836-1398-27.1.26

[42] Pons, D.J., Pons, A.D. and Pons, A.J. (2014) Applied Physics Research, 6, 50-63. http://dx.doi.org/10.5539/apr.v6n3p50

[43] Pons, D.J., Pons, A.D. and Pons, A.J. (2015) Physics Research International, 2015, Article ID: 651361. http://dx.doi.org/10.1155/2015/651361

[44] Pons, D.J., Pons, A.D. and Pons, A.J. (2015) Journal of Nuclear and Particle Physics, 5, 58-69.

[45] Pons, D.J., Pons, A.D. and Pons, A.J. (2014) Journal of Modern Physics, 5, 1980-1994. http://www.scirp.org/Journal/PaperInformation.aspx?paperID=51921 http://dx.doi.org/10.4236/jmp.2014.517193

[46] Pons, D.J., Pons, A.D., Pons, A.M. and Pons, A.J. (2011) Cordus Conjecture: Part 1.2 Quo Vadis, Photon? http://vixra.org/abs/1104.0017

[47] Einstein, A. (1920) Relativity: The Special and General Theory. Holt, New York.

[48] Pons, D.J. and Pons, A.D. (2013) The Open Astronomy Journal, 6, 77-89. http://dx.doi.org/10.2174/1874381101306010077

[49] Pons, D.J., Pons, A.D. and Pons, A.J. (2013) Applied Physics Research, 5, 23-47. http://dx.doi.org/10.5539/apr.v5n6p23

[50] Pons, D.J., Pons, A.D. and Pons, A.J. (2014) Applied Physics Research, 6, 28-46. http://dx.doi.org/10.5539/apr.v6n2p28

[51] Pons, D.J., Pons, A.D. and Pons, A.J. (2013) Applied Physics Research, 5, 145-174. http://dx.doi.org/10.5539/apr.v5n6p145 